\section{Vitreoschisis in Diabetic Macular Edema}

A recent publication ${ }^{1}$ on OCT imaging of the vitreoretinal interface in 44 patients with diabetic macular edema (DME) described that half had anomalous posterior vitreous detachment (PVD), ${ }^{2}$ with membranes attached to the macula and/or optic disc. There were several images, especially Figure $3 \mathrm{~A}$, which the authors interpreted as a "relatively thick EVi membrane, which is overlying cystoid macular edema and tiny amount of subretinal fluid, splits and continuous with both a detached hyperreflective membrane at the posterior vitreous cortex and a broken and thinner hyperreflective adherent ERM." Without clinicopathologic correlation, this interpretation is mere conjecture. In Figure 2 , the authors claim to see "vascularization "; however, the OCT image does not demonstrate blood vessels, and no histopathology is presented. Regarding Figure 4, the authors state, "The B-mode SD-OCT discloses a full-thickness split in the relatively thin unified $\mathrm{ERM} / \mathrm{PViC}$ membrane coupled with its retraction and rolling. "This is a contradiction in terms, as there cannot be "full-thickness" tissue after it splits, a feature that would have been revealed by histopathologic analysis, had it been performed. Indeed, although the clinical observations of this study may be valid, the interpretation of the findings is flawed due to a lack of clinicopathologic correlation and a failure to appreciate the role of diabetic vitreopathy ${ }^{3}$ in advanced disease.

Figure 1 demonstrates a spectral OCT-SLO image obtained from a patient with macular pucker. The vitreoretinal interface in this patient is nearly identical with that in Figure $3 \mathrm{~A}$ of the aforementioned study, with a clearly visible split in the posterior vitreous cortex, known as "vitreoschisis." ${ }^{4}$ In contrast to the aforementioned study, however, this patient underwent clinicopathologic correlation, and the surgically excised tissue was analyzed histopathologically. The results ${ }^{5}$ confirmed schisis of the posterior vitreous cortex.
Vitreoschisis was first described in proliferative diabetic retinopathy (PDR) by ultrasound ${ }^{6}$ and by histopathology, ${ }^{7}$ where $80 \%$ of eyes had a split in the posterior vitreous cortex. Spectral OCT-SLO studies ${ }^{8}$ have furthermore shown that half of eyes with macular hole and macular pucker have evidence of vitreoschisis. In the aforementioned study of DME, ${ }^{1} 13$ (57\%) of the 23 subjects with anomalous PVD had PDR. This is an important consideration, given the high prevalence of vitreoschisis in this group of patients. Thus, the findings at the vitreoretinal interface in the aforementioned study of $\mathrm{DME}^{1}$ are most likely the manifestation of diabetic vitreopathy ${ }^{3}$ with vitreoschisis. Indeed, Figure $3 \mathrm{~A}$ in their publication is an excellent example of vitreoschisis.

The one noteworthy finding in this study is the detection of vitreopapillary adhesion (VPA) in 19 (83\%) of the 23 eyes with anomalous PVD and DME. Previous studies ${ }^{9}$ have shown a similarly high prevalence of VPA in macular holes, lamellar holes with cysts, and macular pucker with cysts. As previously described, ${ }^{9}$ VPA influences the vector of force on the macula, resulting in outward (centripetal) tangential traction inducing macular holes and cystoid spaces in vitreomaculopathies, perhaps in DME as well. This is important, because it has long been presumed that the cysts in DME are fluid-filled manifestations of exudation. It is likely, however, that in cases with VPA there may be a tractional component inducing cystoid spaces that, as in macular holes (full-thickness and lamellar), are not due to exudation. With respect to the authors' remaining interpretations, we must recognize and appreciate the difference between what is seen and what is reality. In The Allegory of the Cave, Socrates spoke of our sensory experiences as mere shadows on the wall in the cave of our misconceived world. ${ }^{10}$ Appreciating the limitations of our senses, even when enhanced by imaging technology, leads to the conclusion that our view of the world is just a model and as such is suscep-

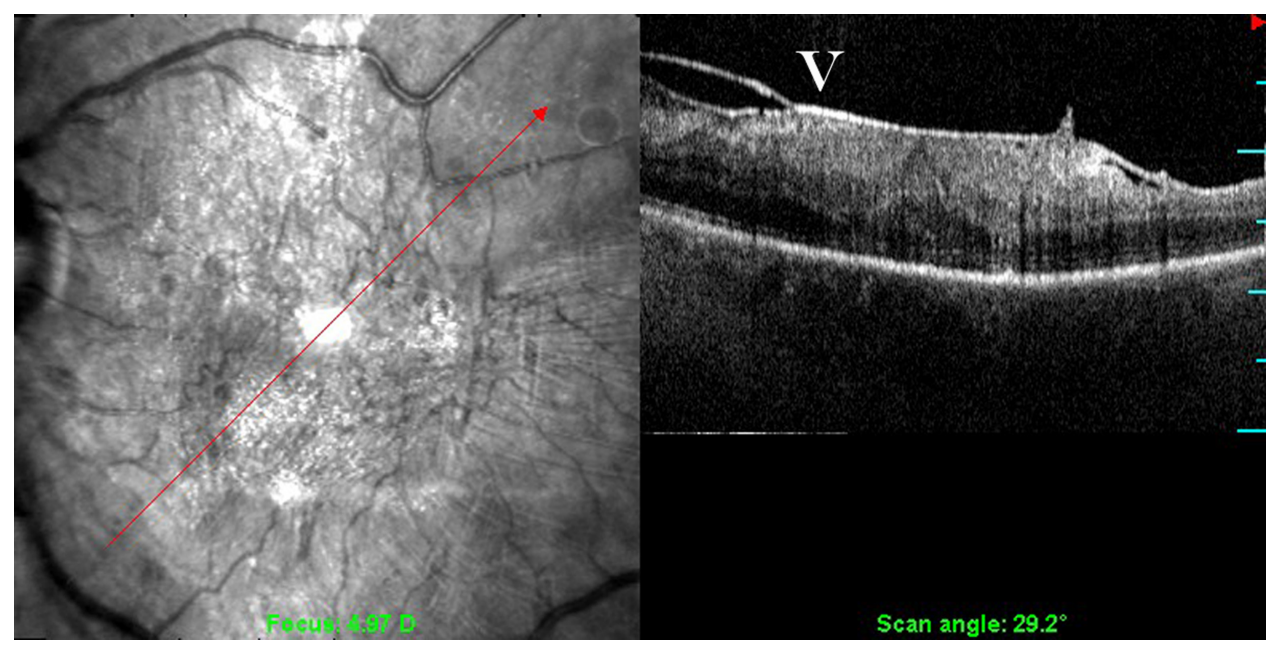

FIGURE 1. Spectral Optical Coherence Tomography (right) combined with Scanning Laser Ophthalmoscopy (left) demonstrates macular pucker in the left eye of a 71-year-old man. Vitreoschisis ("V") is clearly evident in the inferonasal macula. This structure was excised at surgery and histopathologic analysis demonstrated a split in the posterior vitreous cortex with rejoining of the two layers of the split cortex into full-thickness cortex. (Reprinted, with permission, from Gupta et al. Vitreoschisis in macular diseases. $\mathrm{Br} J$ Opbthalmol. 2011;95(3):376-380). 
tible to conditions of context, preconception, and extrapolation. ${ }^{10}$

\section{J. Sebag}

VMR Institute, Huntington Beach, and Doheny Eye Institute, University of Southern California, Los Angeles, California. E-mail: jsebag@VMRinstitute.com

\section{References}

1. Ophir A, Martinez MR. Epiretinal membranes and incomplete posterior vitreous detachment in diabetic macular edema using spectral-domain optical coherence tomography. Invest Ophthalmol Vis Sci. 2011;52:6414-6420.

2. Sebag J. Anomalous PVD. A unifying concept in vitreo-retinal diseases. Graefes Arch Clin Exp Ophthalmol. 2004;242:690 - 698.

3. Sebag J. Diabetic vitreopathy (guest editorial). Ophthalmology. 1996;103:205-206.

4. Sebag J. Vitreoschisis. Graefes Arch Clin Exp Ophthalmol. 2008; 246:329-332

5. Gupta P, Yee KMP, Garcia P, et al. Vitreoschisis in macular diseases. Br J Ophthalmol. 2011;95(3):376-380.

6. Chu TG, Lopez P, Cano MR, et al. Posterior vitreoschisis: an echographic finding in proliferative diabetic retinopathy. Ophthalmology. 1996;103:315-322.

7. Schwartz SD, Alexander R, Hiscott P, et al. Recognition of vitreoschisis in proliferative diabetic retinopathy: a useful landmark in vitrectomy for diabetic traction retinal detachment. Ophthalmology. 1996;103:323-328.

8. Sebag J, Gupta P, Rosen R, Garcia P, Sadun AA. Macular holes and macular pucker: the role of vitreoschisis as imaged by optical coherence tomography/scanning laser ophthalmoscopy. Trans Am Ophthalmol Soc. 2007;105:121-131.

9. Wang MY, Nguyen D, Hindoyan N, Sadun AA, Sebag J. Vitreopapillary adhesion in macular hole and macular pucker. Retina. 2009;29:644-650.

10. Sebag J, Sadun AA. Reflections (guest editorial). Graefes Arch Clin Exp Ophthalmol. 2004;242:811-813.

Citation: Invest Ophthalmol Vis Sci. 2011;52:8455-8456. doi:10.1167/iovs.11-8333

\section{Author Response: Vitreoschisis in Diabetic Macular Edema}

We would like to thank Dr. Sebag for bringing up the issue of vitreoschisis (v-schisis) in diabetic macular edema (DME).

$\mathrm{V}$-schisis is described as splitting within the native multilamellar posterior vitreous cortex $(\mathrm{PViC})$ during an anomalous posterior vitreous detachment (PVD). That definition is similar to that of retinoschisis or iridoschisis, which describe a split in the native relevant tissues. That is different from a split at an hypercellular membrane, which Dr. Sebag and colleagues consider v-schisis as well. ${ }^{1}$ Furthermore, v-schisis that was shown histologically in only one eye in their study, ${ }^{1}$ did not represent a split at the hypercellular ERM, since fibroblasts were not detectable at the specimen. Further, Dr. Sebag wrote in an earlier article, "It is possible that at least part of the multilamellar structure present at the vitreo-retinal interface in pathologic eyes is the internal limiting membrane (ILM) of the retina rather than the PViC alone." 2 This would obviously have a different effect on decision-making of whether a split involves the ILM. Based on the above, when a split is shown by the OCT as occurring within a hyperreflective, fibrocellular epimacular membrane, we refrain from terming it as $\mathrm{v}$-schisis (also explained in the article). Since the split is not at the native $\mathrm{PViC}$, its exact histologic level is unclear, and the mechanism of that split and its consequences might be different from a split at the native PViC. That is in contrast to Dr. Sebag's approach, in which he interprets the membranous split in Figure 1 of his letter as v-schisis.

Posterior vitreous detachment most commonly precedes idiopathic epiretinal membrane (ERM) emergence. ${ }^{3} \mathrm{~V}$-schisis at the native vitreous cortex occurring during an anomalous PVD is hypothesized to be a pathogenesis of less than half of these idiopathic ERMs. In the aforementioned study on idiopathic ERM and macular pucker using the imaging device OCT/scanning laser ophthalmoscopy (SLO), v-schisis that included in definition also eyes with a split at the hypercellular epiretinal membranes associated with the posterior vitreous cortex, was reported to occur in $42 \%(19 / 44)$ of eyes. ${ }^{1}$ However, recent studies did not interpret v-schisis as an association between idiopathic ERM and PViC (or, posterior hyaloid) when the PViC was apparent. For example, in Figure 2 of their study, Koizumi et al. ${ }^{4}$ describe "a hyperreflective zone of the detached posterior hyaloid to be contiguous with the attached posterior hyaloid and the ERM." Chung et al. ${ }^{5}$ also investigated the morphologic features of idiopathic ERMs in the presence or absence of PVD. V-schisis was not referred to anywhere in these manuscripts that studied idiopathic ERMs. Rather, each figure was described as it was actually seen, similarly to the way we describe our observations on the various associations between the ERM and PViC in the DME eyes, including Figure 3A.

In his letter, Dr. Sebag writes in paragraph 3, "the findings [in our study] at the vitreoretinal interface are most likely the manifestation of diabetic vitreopathy with vitreoschisis." First, the words "most likely" direct us to an interpretation. However, we should be attentive to this option, since it might lead us farther from reality, as judged by Socrates and now quoted by Dr. Sebag. Secondly, Dr. Sebag comes to that conclusion by correlating two unrelated data points: (1) $57 \%$ of the 23 subjects in our present study had proliferative diabetic retinopathy (PDR); (2) in another study on PDR, $81 \%$ of eyes were described to have v-schisis. ${ }^{6}$ However, all $81 \%$ of these eyes had either traction retinal detachment or combined traction/rhegmatogenous retinal detachment. This is not the case in our series. Furthermore, in another study on PDR, only $17 \%$ of eyes were suggested by ultrasonography to have v-schisis ${ }^{7}$; however, most of these eyes had vitreous hemorrhage as well. Again, vitreous hemorrhage was not the state in our series. In addition, Dr. Sebag et al. ${ }^{1}$ recently studied the prevalence of v-schisis in various macular diseases using the OCT/SLO and reported the presence of v-schisis in only $13 \%$ of eyes with non-PDR ${ }^{1}$ although without histologic proof in any diabetic eye. Nonetheless, these proposed low prevalences of v-schisis in DME, at $13 \%$ and $17 \%$, as detected by OCT-SLO or ultrasound, respectively, ${ }^{1,7}$ contradict Dr. Sebag's generalization that our findings at the vitreoretinal interface are most likely the manifestation of diabetic vitreopathy with vitreoschisis.

In contrast to idiopathic ERM, the posterior vitreous is commonly attached in DME eyes with ERM. ${ }^{8}$ However, anomalous PVD can occur without v-schisis. ${ }^{3}$ In group A of our DME series ${ }^{9}$ (i.e., incomplete papillary PVD), the vitreous at the macula is attached, so the option of v-schisis at the macula does not seem relevant. Yet it is possible that a variant of a v-schisis process took place in some eyes with partial PVD at the macula (group B), as we explain in the manuscript. However, this should be proven in further studies.

Finally, vascularization associated with the membrane in Figure 2 was detected clinically and is presented now in SD-OCT. Avinoam Ophir
Michael Martinez

Division of Ophthalmology, Hillel-Yaffe Medical Center, Hadera, Israel.

E-mail: ophthalmology@hillel-yaffe.health.gov.il 